\title{
Omega-3 Fatty Acids: Possible Neuroprotective Mechanisms in the Model of Global Ischemia in Rats
}

\author{
Maria Elizabeth Pereira Nobre, ${ }^{1}$ Alyne Oliveira Correia, ${ }^{1}$ \\ Francisco Nilson Maciel Mendonça, ${ }^{1}$ Luiz Ricardo Araújo Uchoa, ${ }^{1}$ \\ Jessica Tamara Nunes Vasconcelos, ${ }^{1}$ Carlos Ney Alencar de Araújo, ${ }^{1}$ \\ Gerly Anne de Castro Brito, ${ }^{2}$ Rafaelly Maria Pinheiro Siqueira, ${ }^{2}$ \\ Gilberto dos Santos Cerqueira, ${ }^{2}$ Kelly Rose Tavares Neves, ${ }^{2}$ \\ Ricardo Mário Arida, ${ }^{3}$ and Glauce Socorro de Barros Viana ${ }^{1,2}$ \\ ${ }^{1}$ Faculty of Medicine, Estácio of Juazeiro do Norte (FMJ), Rua Tenente Raimundo Rocha 515, 63040-360 Juazeiro do Norte, CE, Brazil \\ ${ }^{2}$ Federal University of Ceará (UFC), Rua Coronel Nunes de Melo 1127, 60430-270 Fortaleza, CE, Brazil \\ ${ }^{3}$ Federal University of São Paulo (UNIFESP), Rua Pedro de Toledo 669, 04039-032 São Paulo, SP, Brazil
}

Correspondence should be addressed to Glauce Socorro de Barros Viana; gbviana@live.com

Received 17 December 2015; Revised 1 April 2016; Accepted 4 April 2016

Academic Editor: Duo Li

Copyright ( 2016 Maria Elizabeth Pereira Nobre et al. This is an open access article distributed under the Creative Commons Attribution License, which permits unrestricted use, distribution, and reproduction in any medium, provided the original work is properly cited.

\begin{abstract}
Background. Omega-3 ( $\omega 3$ ) administration was shown to protect against hypoxic-ischemic injury. The objectives were to study the neuroprotective effects of $\omega 3$, in a model of global ischemia. Methods. Male Wistar rats were subjected to carotid occlusion (30 $\mathrm{min})$, followed by reperfusion. The groups were SO, untreated ischemic and ischemic treated rats with $\omega 3(5 \mathrm{and} 10 \mathrm{mg} / \mathrm{kg}, 7$ days). The SO and untreated ischemic animals were orally treated with $1 \%$ cremophor and, $1 \mathrm{~h}$ after the last administration, they were behaviorally tested and euthanized for neurochemical (DA, DOPAC, and NE determinations), histological (Fluoro jade staining), and immunohistochemical (TNF-alpha, COX-2 and iNOS) evaluations. The data were analyzed by ANOVA and Newman-Keuls as the post hoc test. Results. Ischemia increased the locomotor activity and rearing behavior that were partly reversed by $\omega 3$. Ischemia decreased striatal DA and DOPAC contents and increased NE contents, effects reversed by $\omega 3$. This drug protected hippocampal neuron degeneration, as observed by Fluoro-Jade staining, and the increased immunostainings for TNF-alpha, COX-2, and iNOS were partly or totally blocked by $\omega 3$. Conclusion. This study showed a neuroprotective effect of $\omega 3$, in great part due to its antiinflammatory properties, stimulating translational studies focusing on its use in clinic for stroke managing.
\end{abstract}

\section{Background}

Ischemic stroke is a pathologic condition and a major cause of death and disability worldwide. Because of its huge socioeconomic burden and considering the global life expectancy increases, one can assume that stroke is already the most challenging disease [1]. Although animal stroke models have shed light on the pathophysiology of ischemic stroke, the translation of these results from bench to bedside has been somewhat disappointing $[2,3]$.

The most common cause of stroke is the sudden occlusion of a blood vessel by a thrombus or embolism, resulting in an almost immediate loss of oxygen and glucose to the cerebral tissue. Brain is almost exclusively dependent on the continuous steady flow of glucose and oxygen to undergo oxidative phosphorylation for energy production, since it has no energetic stores. Within minutes of vascular occlusion, a complex sequence of pathophysiological events called ischemic cascade occurs. The first consequence is the depletion of substrates, particularly oxygen and glucose, that causes the accumulation of lactate via anaerobic glycolysis. Acidosis may enhance free-radical formation, interfering with intracellular protein synthesis and worsening ischemic brain injury $[4,5]$. Energy failure leads to perturbation of the 
$\mathrm{Na}^{+} / \mathrm{K}^{+}$-ATPases and $\mathrm{Ca}^{2+} / \mathrm{H}$-ATPases pumps and, in addition, the $\mathrm{Na}^{+}-\mathrm{Ca}^{2+}$ transporter is reversed [6]. Subsequently, an alteration of ion homeostasis causes cytotoxic edema, leading to events triggered by intracellular $\mathrm{Ca}^{2+}$ excess [1].

$\omega 3$ fatty acids are a group of essential fatty acids that serve as energy substrates and integral membrane components and, therefore, play crucial roles in the maintenance of normal neurological functions. Recent studies show that $\omega 3$ fatty acids display neuroprotective properties and may exert beneficial effects on cerebral ischemia and other brain disorders [7]. It is well recognized that cerebral ischemia induces excessive release of excitatory amino acids, as glutamate and aspartate, which provoke enzymatic processes leading to irreversible neuronal injury [8]. On the other hand, several years ago, the behavioral effects of $\omega 3$ fatty acids deficiency were proposed to be mediated through monoaminergic neurotransmission, including the dopaminergic system, what was evidenced years later $[9,10]$.

Thus, the objectives of the present work were to study the possible neuroprotective effects of $\omega 3$ fatty acids in a model of global brain ischemia in rats, focusing on behavior and striatal DA, DOPAC, and NE levels. Besides, considering that inflammation plays an important role in the pathogenesis of ischemic stroke $[11,12]$ and the anti-inflammatory properties of $\omega 3$ [13-16] also observed by us [17], we decided to analyze the action of $\omega 3$ fatty acids on proinflammatory cytokines and enzymes, in hippocampi from ischemic animals, through TTC and Fluoro-Jade staining and with immunohistochemical assays.

\section{Material and Methods}

2.1. Drugs and Reagents. Proepa (Aché Laboratórios Farmacêuticos SA) was the source of $\omega 3$ fatty acids, while sodium thiopental was from Laboratório Cristália, Brazil. Cremophor EL, 2,3,5-triphenyltetrazolium chloride (TTC), standard monoamines, and standard amino acids were from Sigma-Aldrich, USA. All other reagents were of analytical grade.

2.2. Animals. Male Wistar rats $(200-250 \mathrm{~g})$ were obtained from the Animal House of the Faculty of Medicine Estácio of Juazeiro do Norte, Brazil. The animals were housed at $24 \pm$ $2^{\circ} \mathrm{C}$, under a $12 \mathrm{~h}$ light $/ 12 \mathrm{~h}$ dark cycle and had free access to a standard pellet diet (Purina chow) and tap water. They were deprived of food for $8 \mathrm{~h}$, before the experiments, except for drinking water. The animals were treated in accordance with the current law and the NIH Guide for the Care and Use of Laboratory Animals. The project was previously approved by the Animal's Ethics Committee of the Faculty of Medicine of the Federal University of Ceará at the city of Barbalha, Brazil.

2.3. Experimental Protocol. The animals were anesthetized with sodium thiopental $(50 \mathrm{mg} / \mathrm{kg}$, i.p.) and submitted to the model of transitory global ischemia, by the bilateral occlusion of both carotids, for $30 \mathrm{~min}$. After reperfusion, the incision was sutured and the animals placed in individual plastic cages for recovery, with water and food ad libitum. The shamoperated groups (SO, controls) were submitted to the same procedure, except for the clamping of the carotids. After surgery, the animals were orally treated (by gavage) with $\omega 3$ fatty acids, at the doses of 5 and $10 \mathrm{mg} / \mathrm{kg}$, daily for 7 days. The SO and ischemic groups received 1\% Cremophor $(1 \mathrm{~mL} / \mathrm{kg})$. The animals were distributed into 4 groups (except for COX-2 immunostaining, where the $\omega 3$ dose of $2.5 \mathrm{mg} / \mathrm{kg}$ was also used) as follows: SO, untreated ischemic, and ischemic groups after treatments with $\omega 3$ ( 5 and $10 \mathrm{mg} / \mathrm{kg}$, p.o.). At the 7 th day of treatment and $1 \mathrm{~h}$ after the last drug administration, the animals were submitted to behavioral tests (open field and water maze tests) and sacrificed for striata dissection and hippocampal slicing. The striata were used for homogenate preparations and DA, DOPAC, and $\mathrm{NE}$ determinations. Hippocampal slices were processed for immunohistochemistry assays.

\subsection{Behavioral Testing}

2.4.1. Open Field Test. This test is used to measure locomotor activity in rodents and can also serve to determine motor deficits and anxiety. It was originally described for the study of emotionality in rats and now is one of the most popular models in animal psychology [18]. Locomotor activity is measured by determining the amount of distance traveled and stereotyped behaviors, as rearing and grooming. The test is sensitive to motor dysfunction, as well as hippocampal and basal ganglia damage, and we used an arena $(50 \times 50 \times 20 \mathrm{~cm})$ divided into four equal quadrants. Each animal was evaluated for $5 \mathrm{~min}$, at the 7th day after ischemia, and we determined the number of quadrants crossed by the animal with all 4 paws.

2.4.2. Morris Water Maze Test. This is a test of spatial learning for rodents that relies on distal cues to navigate from start locations around the perimeter of an open swimming arena for locating a submerged escape platform. The original procedure was described as Morris water maze [19, 20]. Spatial learning is assessed across repeated trials and reference memory. The test has proven to be a robust and reliable one and is strongly correlated with hippocampal synaptic plasticity and NMDA receptor function. For that, we used a circular black pool (1.7 m of diameter and $1 \mathrm{~m}$ height), filled with water $(0.59 \mathrm{~m}$ deep $)$, at $25^{\circ} \mathrm{C}$ temperature. The pool is divided into four quadrants and has a $10 \mathrm{~cm}$ diameter platform immersed $0.5 \mathrm{~cm}$ below the water surface. The room is provided with four cues located on North, South, East, and West of the walls. The animals were subjected to two trials, for two consecutive days (pretraining), and, $48 \mathrm{~h}$ after the last trial, to the test in the water maze. The animals had a maximum time of $54 \mathrm{~s}$ (cut-off time) for finding the platform, where they can stay for $15 \mathrm{~s}$ but would have a second chance, in case of failure.

2.4.3. Catecholamine Determinations by HPLC. For measurements of dopamine (DA) and its metabolite (3,4-dihydroxyphenylacetic acid, DOPAC) as well as noradrenaline (NE), striata from all groups were used to prepare $10 \%$ homogenates. Homogenates were sonicated in $0.1 \mathrm{M} \mathrm{HClO}_{4}$, for $30 \mathrm{~s}$, and centrifuged at $4^{\circ} \mathrm{C}$ for $15 \mathrm{~min}$ at $15,000 \mathrm{rpm}$, and the supernatants were filtered $(0.2 \mu \mathrm{m}$, Millipore). 
Twenty-microliter samples were then injected into a highperformance liquid chromatograph (HPLC) column. The mobile phase was $0.163 \mathrm{M}$ citric acid, $\mathrm{pH}$ 3.0, containing $0.02 \mathrm{mM}$ EDTA with $0.69 \mathrm{mM}$ sodium octanesulfonic acid (SOS), as an ion pairing reagent, $4 \% \mathrm{v} / \mathrm{v}$ acetonitrile, and $1.7 \%$ $\mathrm{v} / \mathrm{v}$ tetrahydrofuran. The monoamines were electrochemically detected, using an amperometric detector (Shimadzu, Japan), by oxidation on a glassy carbon electrode at $0.85 \mathrm{~V}$ relative to the $\mathrm{Ag}-\mathrm{AgCl}$ reference electrode. Their concentrations were determined by comparison with standards injected into the HPLC column at the day of experiment and the values expressed as $\mathrm{ng} / \mathrm{g}$ tissue.

2.4.4. TTC Staining. TTC staining is considered a reliable method for detection of cerebral infarction in rats after ischemia [21]. The animals were decapitated and their brains removed for TTC (2,3,5-triphenyltetrazolium chloride) staining. Then, $2 \mathrm{~mm}$ coronal sections were obtained with the rodent brain matrix apparatus (Harvard, USA). The slices were incubated with TTC for $30 \mathrm{~min}$ at $37^{\circ} \mathrm{C}$ in the dark and fixed by immersion in $4 \%$ buffered (7.4) formaldehyde solution for $24 \mathrm{~h}$. Afterwards, the sections were photographed and ischemic areas were quantified by the Image J software (NIH, USA). In those cells populations where the electron mitochondrial transport is maintained, the TTC shows a red color that is greatly decreased in brain ischemic areas.

2.4.5. Fluoro-Jade Staining. Fluoro-Jade is an anionic fluorescein derivative, used for the histological staining of neurons which become fluorescent after degeneration. After paraffin removal (by immersion in xylol), hippocampus sections $(5 \mu \mathrm{m})$ were mounted on slides surrounded by gelatin and rehydrated by immersion in ethanol for $3 \mathrm{~min}$, followed by immersions in 70 and 50\% ethanol solutions and distilled water. The slices were placed into a $0.06 \%$ potassium permanganate solution, for $15 \mathrm{~min}$, washed in distilled water, and transferred to a Fluoro-Jade solution where they stayed for $30 \mathrm{~min}$ (with gentle stirring). After staining, the slices were washed in distilled water ( 3 times, 2 min each time). The excess of water was discarded and the dry slices mounted in Fluoromount ${ }^{\circledR}$ media and examined with a fluorescence microscope. The data were quantified by the Image J software (NIH, USA).

2.4.6. Immunohistochemistry Assays for TNF-Alpha, COX-2, and $i N O S$. Brain hippocampal sections were fixed in $10 \%$ buffered formol, for $24 \mathrm{~h}$, followed by a $70 \%$ alcohol solution and embedded into paraffin wax for slices processing on appropriate glass slides. These were placed into the oven at $58^{\circ} \mathrm{C}$, for $10 \mathrm{~min}$, followed by deparaffinization in xylol, rehydration in alcohol at decreasing concentrations, and washing in distilled water and PBS (0.1 M sodium phosphate buffer, $\mathrm{pH}$ 7.2), for $10 \mathrm{~min}$. The endogenous peroxidase was blocked with a $3 \%$ hydrogen peroxide solution, followed by incubation with the appropriate primary anti-antibody for TNF-alpha, iNOS, and COX-2, and diluted according to the manufacturers' instructions (Santa Cruz or Millipore, USA), for $2 \mathrm{~h}$, at room temperature in a moist chamber. The glass slides were then washed with PBS ( 3 times, 5 min each) and incubated with the biotinylated secondary antibody, for $1 \mathrm{~h}$, at room temperature in a moist chamber. Then, they were washed again in PBS and incubated with streptavidinperoxidase, for $30 \mathrm{~min}$, at room temperature (in a moist chamber) and, after a final wash in PBS, incubated in $0.1 \%$ $\mathrm{DAB}$ solution (in 3\% hydrogen peroxide). Finally, the glass slides were washed in distilled water and counterstained with Mayer's hematoxylin, washed in tap water, dehydrated in alcohol (at increasing concentrations), diaphonized in xylol, and mounted on Entelan ${ }^{\circledR}$ for optic microscopy examination. The data were quantified by the Image J software (NIH, USA).

2.5. Statistical Analyses. The data are presented as means \pm SEM and analyzed by one-way ANOVA, followed by Newman-Keuls test as the post hoc test. Whenever needed the data were analyzed by two-tailed unpaired Student's $t$ test. The differences were considered statistically significant at $p<0.05$.

\section{Results}

\subsection{Behavioral Tests}

3.1.1. Open Field Test. We showed a 1.9-fold increase in locomotor activity in the untreated ischemic group, as related to the sham-operated one (SO, controls). The treatment of ischemic groups with $\omega 3$ (5 and $10 \mathrm{mg} / \mathrm{kg}$, p.o.) significantly reversed the locomotor activity to values close to normality (Figure 1(a)). A similar profile was seen with the rearing behavior (Figure 1(b)). These effects were reversed after $\omega 3$ treatments and, in both cases, the values were even lower than those of the sham-operated group.

3.1.2. Morris Water Maze Test. We showed a significant 2 -fold increase in the time to find the platform after ischemia, as related to controls (SO), indicating an impairment of spatial learning and hipocampal dysfunction. On the other hand, the repeated treatment of ischemic groups with $\omega 3$ ( 5 and $10 \mathrm{mg} / \mathrm{kg}$ ), for 7 days, completely reversed the effects, showing an improvement on spatial memory due to $\omega 3$ treatments (Figure 2).

\subsection{Neurochemical Determinations}

3.2.1. Dopamine (DA), DOPAC, and NE Determinations in Rat Brain Striatum. Our results showed that brain ischemia decreases in 37\% DA levels, as related to controls (SO) (Figure 3(a)). On the other hand, DA values returned to normality (and similarity to those of the SO group), in the ischemic group treated for 7 days with $\omega 3(5$ and $10 \mathrm{mg} / \mathrm{kg}$, p.o.). Similar results were observed with DOPAC where a $26 \%$ decrease was seen in the ischemic untreated group, as related to controls (SO). In both cases, the values in the ischemic group after $\omega 3$ treatments (Figure $3(\mathrm{~b})$ ) returned to those shown by the SO group. Surprisingly, a 9-fold increase in $\mathrm{NE}$ contents, as related to the SO group, was observed in the untreated ischemic group. NE levels returned to values even 


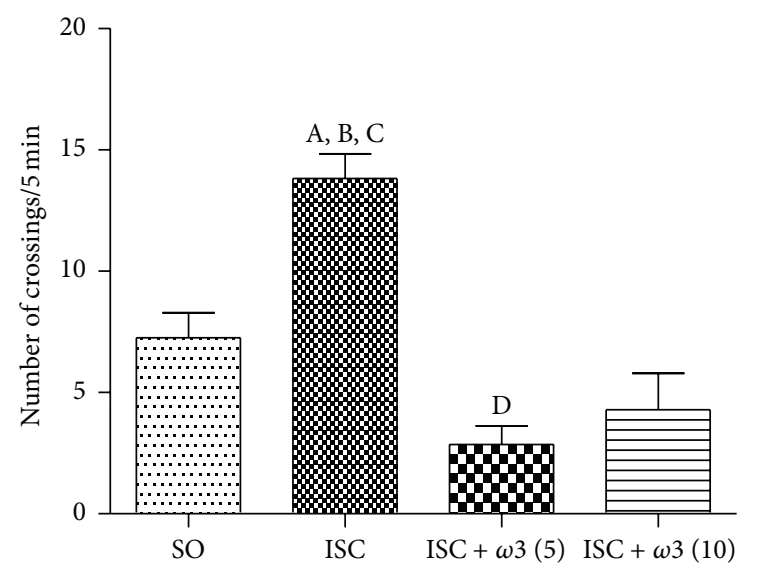

(a)

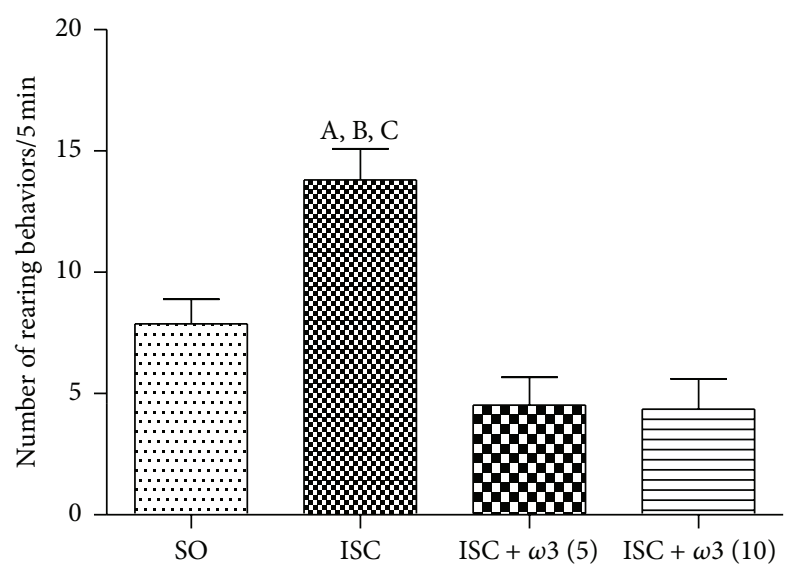

(b)

FIGURE 1: Evaluations of the number of crossing (a) and rearing (b) behaviors by the open field test. The columns represent means \pm SEM (number of crossings $/ 5 \mathrm{~min}$ ) from SO and rats subjected to cerebral ischemia untreated or after oral treatments with $\omega 3$, at the doses of 5 and $10 \mathrm{mg} / \mathrm{kg}$ (number of animals per group: $\mathrm{SO}=27$; ISC $=35$; ISC $+\omega 3(5)=14 ; \mathrm{ISC}+\omega 3(10)=17)$. (a) (A) versus $\mathrm{SO}, \mathrm{q}=6.591^{* * *} ;(\mathrm{B})$ versus ISC $+\omega 3(5), q=8.916^{* * *} ;(C)$ versus ISC $+\omega 3(10), q=8.288^{* * *} ;(D)$ versus $\mathrm{SO}, \mathrm{q}=3.435^{*}$. (b) (A) versus SO, $\mathrm{q}=5.533^{* * *}$; (B) versus ISC $+\omega 3(5), q=6.808^{* * *} ;(C)$ versus ISC $+\omega 3(10), q=7.246^{* * *}$ (one-way ANOVA and Newman-Keuls test as the post hoc test).

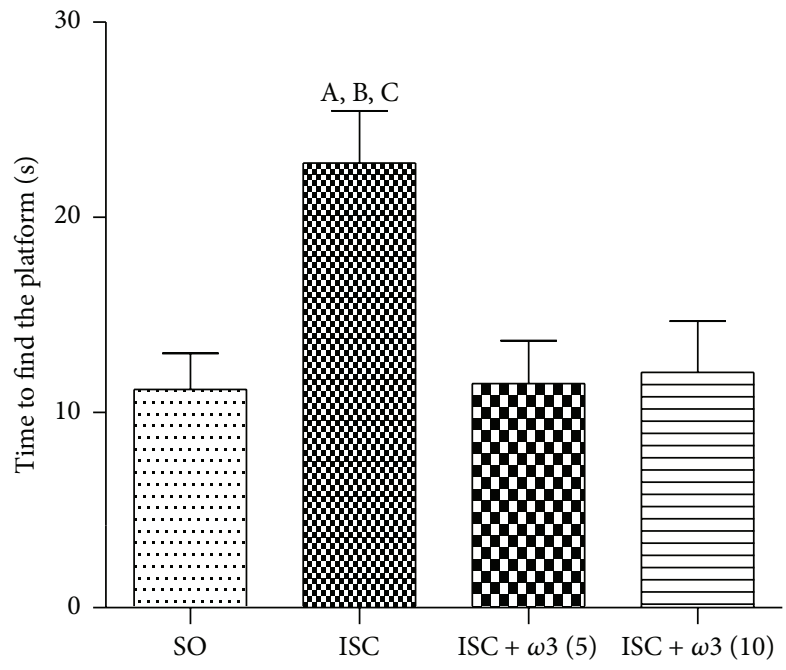

FIGURE 2: Evaluation of the spatial memory by the water maze test. The columns represent means \pm SEM of the time $(s)$ to find the platform. The studied groups were SO and ischemic animals untreated or after oral treatments with $\omega 3$, at the doses of 5 and $10 \mathrm{mg} / \mathrm{kg}$ (number of animals per group: $\mathrm{SO}=28 ; \mathrm{ISC}=27 ; \mathrm{ISC}+\omega 3$ $(5)=14 ; \mathrm{ISC}+\omega 3(10)=14)$. (A) versus $\mathrm{SO}, \mathrm{q}=5.159^{* *} ;(\mathrm{B})$ versus $\omega 3(5), q=4.016^{*}$; (C) versus $\omega 3(10), q=3.897^{* *}$ (one-way ANOVA and Newman-Keuls test as the post hoc test).

lower than those of the SO group, in ischemic animals after $\omega 3$ treatments with the doses of 5 and $10 \mathrm{mg} / \mathrm{kg}$ (Figure 3(c)).

\subsection{Histological and Immunohistochemistry Assays}

3.3.1. TTC Staining in the Hippocampus. The ischemic group (ISC) showed a 69\% decrease in TTC staining as related to the SO group. This decrease was of only $13 \%$ in the ischemic group after $\omega 3$ treatment with the dose of $10 \mathrm{mg} / \mathrm{kg}$
$($ ISC $+\omega 3(10))$. On the other hand, no significant differences were observed in the ISC group (ISC) without and after treatment with $\omega 3$ at the dose of $5 \mathrm{mg} / \mathrm{kg}$ (ISC $+\omega 3(5)$ ) (Figure 4).

3.3.2. Fluoro-Jade Staining in the Hippocampus. A greater number of fluorescent cells were observed in the CA1 hippocampal subfield of the untreated ischemic group, indicating neuronal degeneration. This change is quantified by the Image J software as a decrease (50\%) in optical density, as related to the SO group whose neurons appear darker. A similar picture was observed in the CA3 area (51\% decrease) and in the dentate gyrus (56\% decrease). In all cases, these alterations were completely reversed after $\omega 3$ treatments (Figure 5).

3.3.3. Immunohistochemistry for TNF-Alpha, COX-2, and iNOS in Hippocampus and Temporal Cortex. The immunohistochemistry data for TNF-alpha showed a higher number of immunopositive cells in CA1, CA3, and dentate gyrus areas in the ischemic group (ISC) as related to the SO group. The effects were much more intense in the CA1 (313fold increase), followed by DG (30-fold increase) and CA3 (14-fold increase) areas. In all cases, these changes were significantly decreased after $\omega 3$ treatments (Figure 6).

Figure 7 shows representative photomicrographs for COX-2 immunohistochemistry assays in the dentate gyrus (DG) and hilus. While increases of 6- and 5-fold were demonstrated in the DG and hilus ischemic groups, respectively, these effects were significantly reduced to similar values in both areas after $\omega 3$ treatments. Increases around 3.9-fold in the iNOS immunoreactivity were detected in the CA3 area from ischemic groups as related to the $\mathrm{SO}$ group. The effects were reduced to values even lower than those from the SO group after treatments with $\omega 3$ at the dose of $5 \mathrm{mg} / \mathrm{kg}$ (Figure 8). 


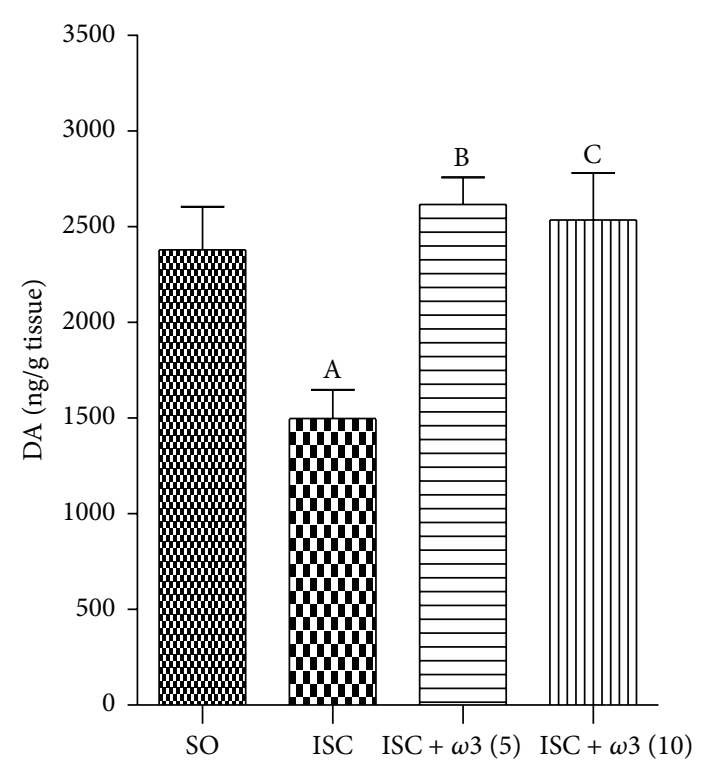

(a)

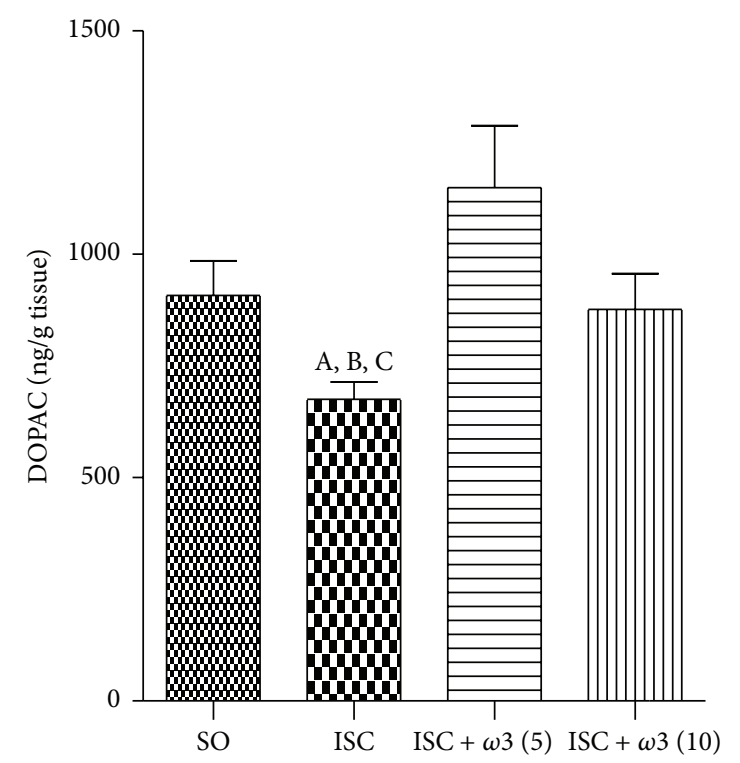

(b)

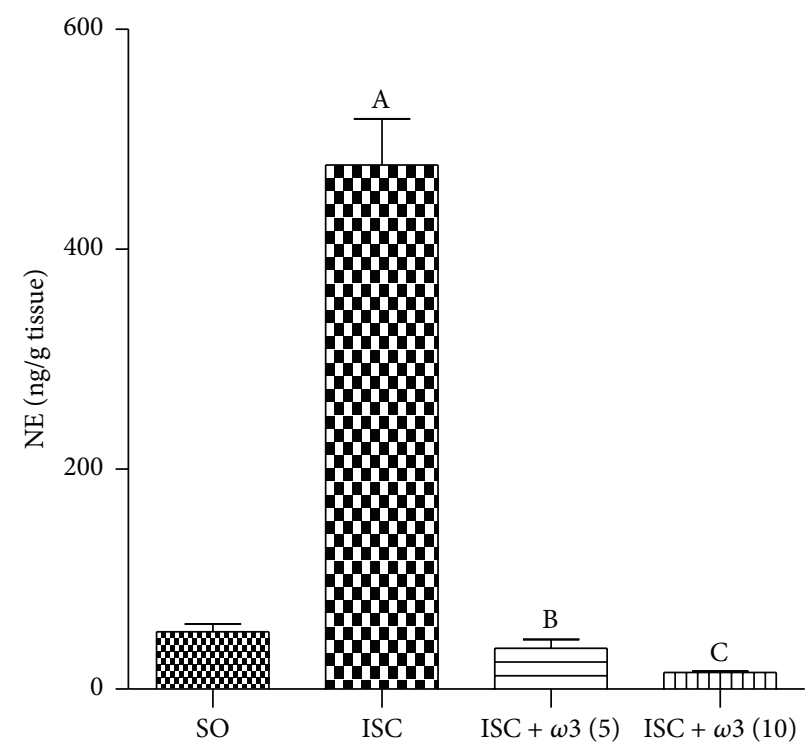

(c)

FIGURE 3: Evaluation of $\omega 3$ treatments at the doses of 5 and $10 \mathrm{mg} / \mathrm{kg}$ on the striatal contents of DA (a), DOPAC (b), and NE (c), in rats subjected to brain ischemia and reperfusion for 7 days. The numbers of animals per group were the following: for DA, $S O=14$, ISC $=9$, ISC $+\omega 3(5)=19$, and ISC $+\omega 3(10)=15$; for DOPAC, $S O=14$, ISC $=11$, ISC $+\omega 3(5)=15$, and ISC $+\omega 3(10)=14 ;$ for NE, SO $=8$, ISC $=10$, ISC $+\omega 3(5)=8$, and ISC $+\omega 3(10)=6$. The columns represent means \pm SEM and the groups are sham-operated (SO); ischemic (ISC) untreated; and ischemic after $\omega 3$ treatments for 7 days. DA: $(A)$ versus $S O, q=3.830^{* *} ;(B)$ versus $\omega 3(5), q=5.145^{* *} ;(C)$ versus $\omega 3(10), q=4.570^{* *}$. DOPAC: $(A)$ versus $S O, t=2.484, d f=23 ;(B)$ versus $\omega 3(5), q=4.707^{* *} ;(C)$ versus $\omega 3(10), t=2.085, d f=23$. NE: $(A)$ versus $S O, q=16.52^{* * *}$; (B) versus $\omega 3$ (2.5), $q=18.79^{* * *}$; (C) versus $\omega 3$ (5), $q=17.09^{* * *}$ (one-way ANOVA and Newman-Keuls test as the post hoc test).

\section{Discussion}

Global cerebral ischemia in rodents is characterized morphologically by a selective neuronal damage, particularly in the hippocampus, but also in the striatum and cortex [22]. The resultant damage to vulnerable cells, notably in the CA1 and hilar hippocampal fields, is frequently associated with memory deficits [23]. The most common model for global cerebral ischemia in rodents uses brain ischemia associated with hypotension [24], which results in higher brain damage mainly in the hippocampus. However, in the present work we used the common carotids occlusion for $30 \mathrm{~min}$ without hypotension, which has been shown to cause alterations in hippocampal CA1 neurons [25].

Furthermore, animal studies particularly in rodents indicate that long chain $\omega 3$ fatty acids play a role in behavioral and cognitive functions. Chronic $\omega 3$ fatty acids deficiency has been shown to increase anxiety in rodents, particularly under stressful conditions [26-28]. It has been demonstrated that a decreased content of docosahexaenoic acid (DHA), a long 

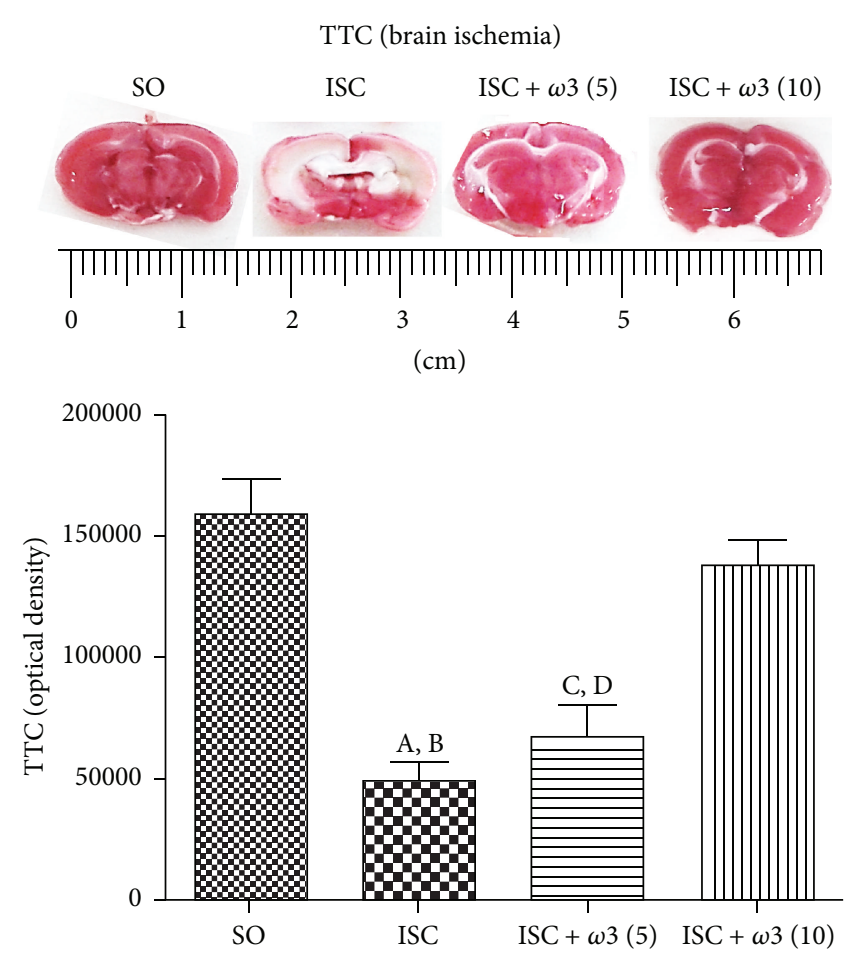

FIGURE 4: Effects of $\omega 3$ treatments in ischemic groups, visualized by the 2,3,5-triphenyltetrazolium chloride (TTC) staining as related to the SO group. (A) versus SO, $\mathrm{q}=9.546^{* * *}$; (B) versus ISC + $\omega 3(10), \mathrm{q}=8.194^{* * *} ;(\mathrm{C})$ versus $\mathrm{SO}, \mathrm{q}=7.894^{* * *}$; (D) versus ISC + $\omega 3$ (10), $\mathrm{q}=$ $6.452^{* * *}$ (one-way ANOVA and Newman-Keuls test as the post hoc test).
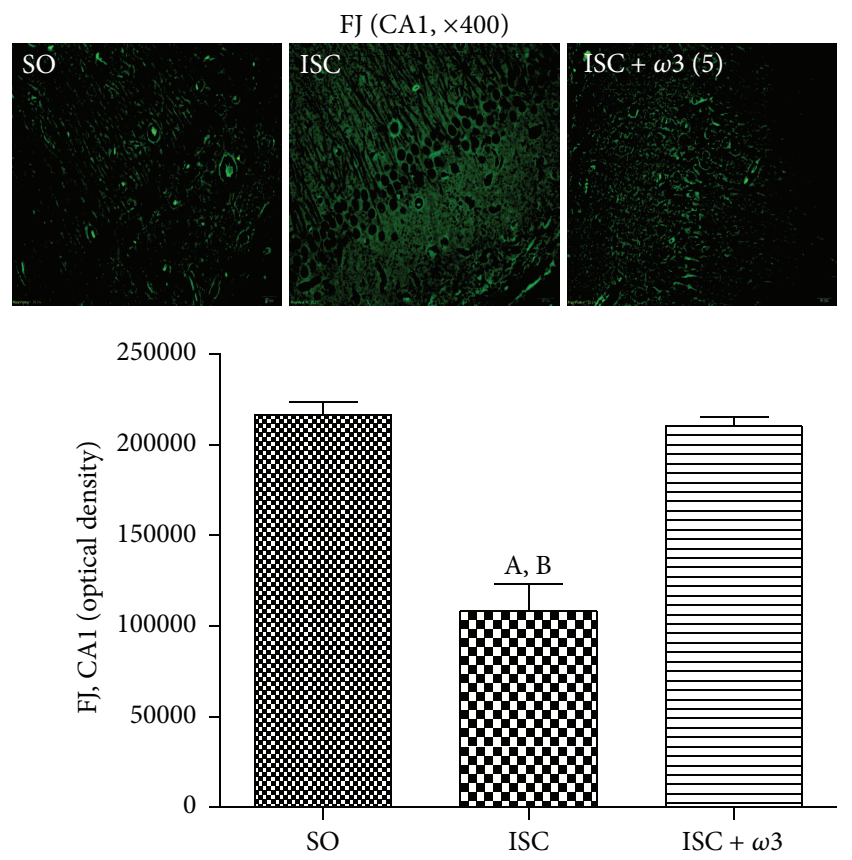

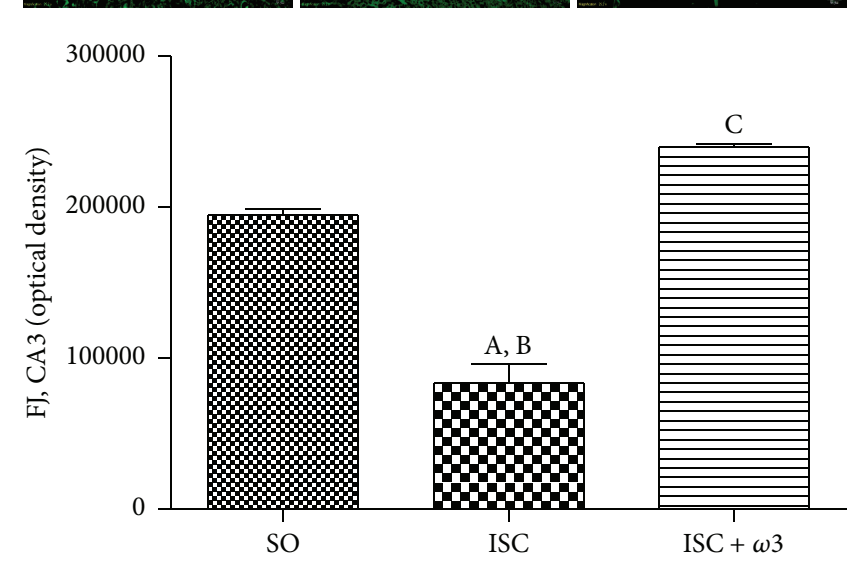

FJ $(\mathrm{CA} 3, \times 400)$
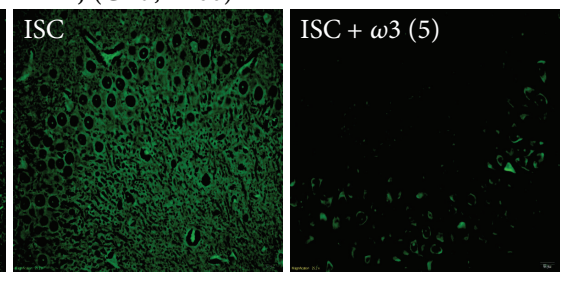

FIGURE 5: $\omega 3$ treatments of ischemic animals (3 animals per group) drastically decreased the neuronal degeneration (visualized by an intense fluorescence to Fluoro-Jade) in hippocampus and cortex. The effects in CA1 and CA3 and DG are represented by photomicrographs and quantitative measurements performed with the Image J software, from 3 to 6 fields. The groups are sham-operated (SO); ischemic (ISC) untreated, and ischemic after $\omega 3$ treatments $\left(5 \mathrm{mg} / \mathrm{kg}\right.$, for 7 days). CA1: (A) versus $\mathrm{SO}, \mathrm{q}=10.98^{* * *}$; (B) versus ISC $+\omega 3, \mathrm{q}=10.34^{* * * *}$. CA3: (A) versus $\mathrm{SO}, \mathrm{q}=14.57^{* * *}$; (B) versus ISC $+\omega 3, \mathrm{q}=20.55^{* * *} ;(\mathrm{C})$ versus $\mathrm{SO}, \mathrm{q}=5.979^{* * *}$ (one-way ANOVA and Newman-Keuls test as the post hoc test). 
TNF-alpha $(\mathrm{CA} 1, \times 100)$
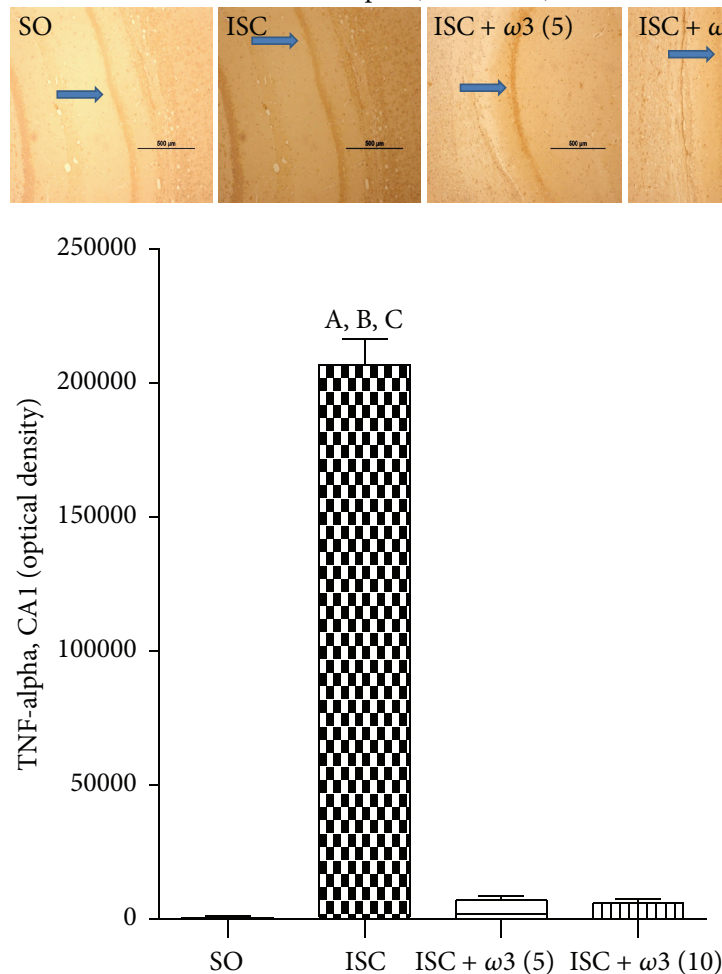

TNF-alpha $(\mathrm{CA} 3, \times 100)$
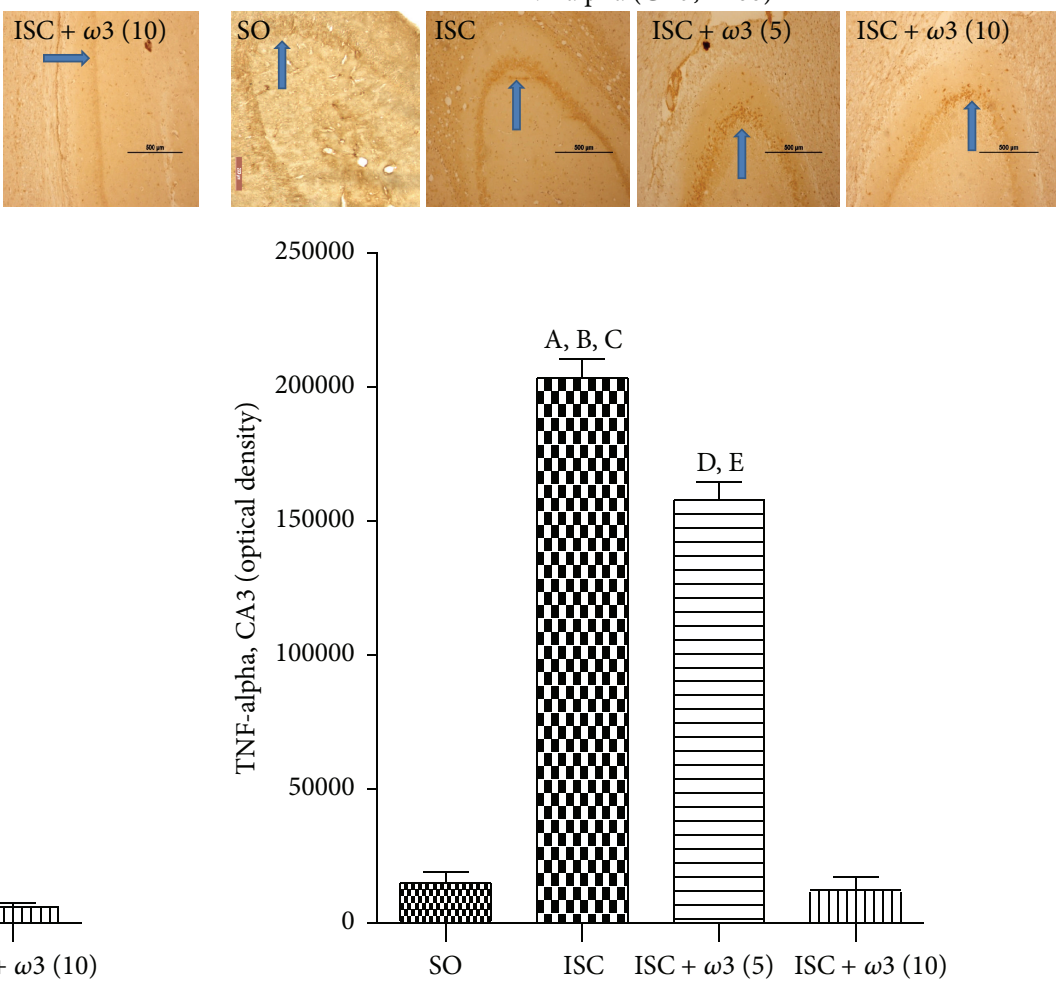

TNF-alpha $(\mathrm{DG}, \times 400)$
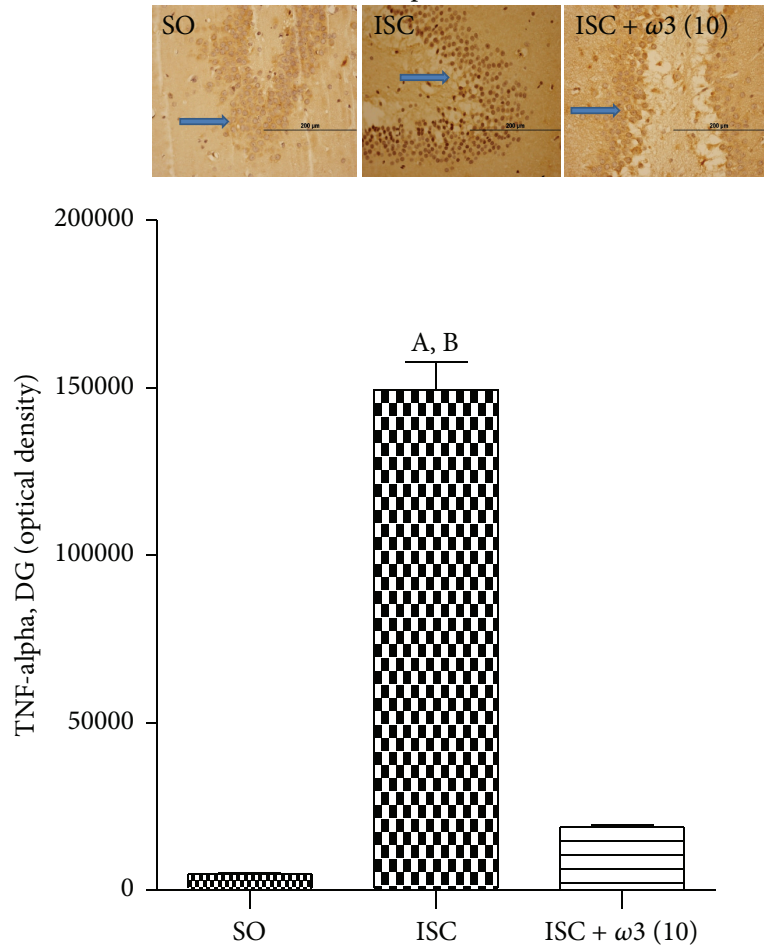

FIGURE 6: $\omega 3$ treatments of ischemic animals ( 3 animals per group) reduced the immunostaining for TNF-alpha, mainly in CA1 and CA3 hippocampal subfields and in the dentate gyrus (DG). Representative photomicrographs and quantitative measurements performed with the Image J software from 3 to 5 fields. The groups are SO, untreated ischemic (ISC) and ischemic after $\omega 3$ treatments (5 and $10 \mathrm{mg} / \mathrm{kg}, 7 \mathrm{days}$ ). CA1: (A) versus SO, $\mathrm{q}=41.76^{* * *}$; (B) versus ISC $+\omega 3(5), \mathrm{q}=40.44^{* * *} ;(\mathrm{C})$ versus ISC $+\omega 3(10), \mathrm{q}=40.63^{* * *}$. CA3: (A) versus SO, $\mathrm{q}=$ $32.86^{* * *} ;(\mathrm{B})$ versus ISC + $\omega 3(5), \mathrm{q}=7.494^{* * *} ;(\mathrm{C})$ versus ISC $+\omega 3(10), \mathrm{q}=33.31^{* * *} ;(\mathrm{D})$ versus SO, $\mathrm{q}=7.494^{* * *} ;(\mathrm{E})$ versus ISC $+\omega 3(10)$, $\mathrm{q}=23.91^{* * *}$. DG: $(\mathrm{A})$ versus $\mathrm{SO}, \mathrm{q}=30.26^{* * *} ;(\mathrm{B})$ versus ISC $+\omega 3(10), \mathrm{q}=27.34^{* * *}$ (one-way ANOVA and Newman-Keuls test as the post hoc test). 

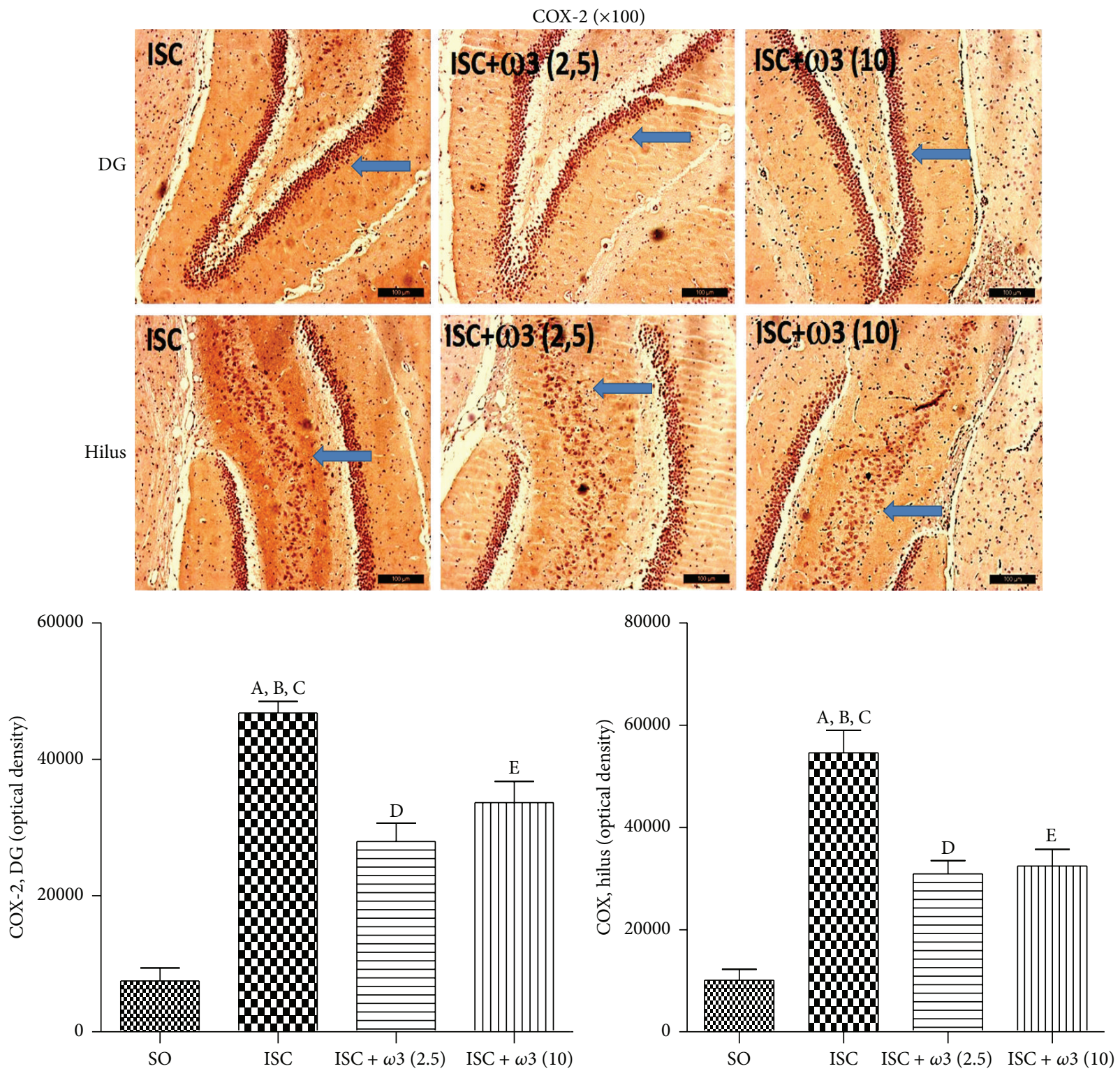

FIGURE 7: $\omega 3$ treatments of ischemic animals (3 animals per group) reduced the immunostaining for COX-2, mainly in the dentate gyrus (DG) and hilus. Representative photomicrographs and quantitative measurements performed with the Image J software, from 3 to 5 fields. The groups are untreated ischemic (ISC) and ischemic after treatments with $\omega 3$ ( 2.5 and $10 \mathrm{mg} / \mathrm{kg}, 7$ days). DG: (A) versus SO, q $=14.88^{* * *}$; (B) versus ISC $+\omega 3(2.5), q=7.135^{* * *}$; (C) versus ISC $+\omega 3(10), q=4.979^{* *}$. Hilus: (A) versus SO, $q=13.95^{* * *}$; (B) versus ISC $+\omega 3$ (2.5), $\mathrm{q}=7.418^{* * *} ;(\mathrm{C})$ versus ISC $+\omega 3(10), \mathrm{q}=6.943^{* * *} ;$ (D) versus SO, $\mathrm{q}=6.527^{* * *}$; (E) versus SO, $\mathrm{q}=7.003^{* * *}$ (one-way ANOVA and Newman-Keuls test as the post hoc test).

chain polyunsaturated fatty acid of the $\omega 3$ series, is accompanied by anxiety and learning and memory impairments that have been associated with changes in neurotransmission processes [10].

Most of the literature data deal with animals chronically submitted to $\omega 3$ supplemented diet [29-33] or $\omega 3$ high dose, as seen in preclinical $[34,35]$ and clinical studies as well [36]. As far as we know, ours is the first study dealing with $\omega 3$ administration for a short period and at a lower dose range. We focused on behavioral changes, determinations of striatal monoamines, and immunohistochemistry assays in the hippocampus of animals submitted to global brain ischemia untreated or treated with $\omega 3$ fatty acids.

The majority of studies performed with $\omega 3$ describe its dietary effects after 3 to 5 weeks [37-39] and very few [40] use a short-term treatment (1 week). While this last study showed an effect of $\omega 3$ on lipid metabolism after shortterm treatment, others [41] observed no effect after the $\omega 3$ treatment for 2 weeks on the scopolamine-induced amnesia. In the present study we demonstrated that $\omega 3$ at lower doses can be effective after daily administration by gavage to rats and well absorbed after its emulsification as already shown [42] and in an empty stomach. This short-term effect of lower 


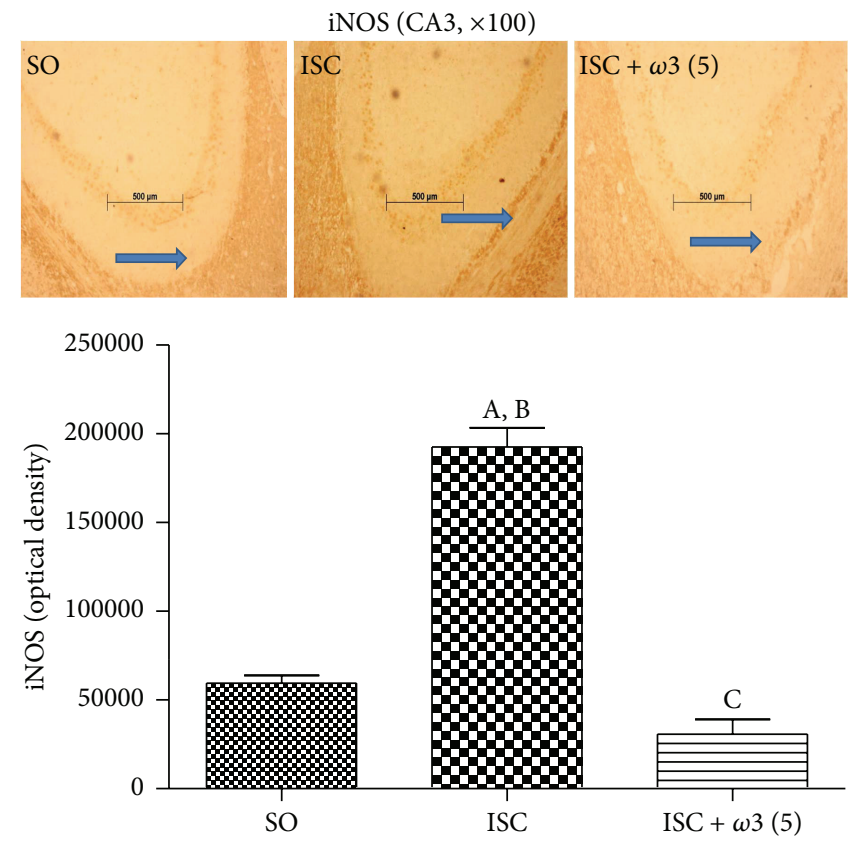

FIGURE 8: $\omega 3$ treatments ( 3 animals per group) reduced the immunostaining for iNOS mainly in the CA3 hippocampal subfield. Representative photomicrographs and measurements performed with the Image J software, from 3 to 5 fields. The groups are sham-operated (SO), untreated ischemic (ISC), and ischemic after $\omega 3$ treatment $\left(5 \mathrm{mg} / \mathrm{kg}, 7\right.$ days). (A) versus $\mathrm{SO}, \mathrm{q}=16.03^{* * *}$; (B) versus ISC $+\omega 3(5), \mathrm{q}=19.49^{* * *} ;(\mathrm{C})$ versus $\mathrm{SO}, \mathrm{q}=3.456^{*}$ (one-way ANOVA and Newman-Keuls test as the post hoc test).

doses of $\omega 3$ was clearly observed by us after the TTC staining, a reliable method for the detection of brain ischemic areas in the rat [21].

In the open field test, we showed that after global brain ischemia the animals presented not only an increased locomotor activity, but also an increased number of rearing behaviors, as related to controls ( $\mathrm{SO}$ ). On the other hand, the treatment of ischemic groups with $\omega 3$ significantly decreased all these behavioral parameters to values close to those of controls. Brain ischemia is known to increase locomotor activity and anxiety [43-47] and the degree of hippocampal damage has been positively correlated with the increase in motor activity.

Our data showed that brain ischemia significantly impaired spatial memory, and this effect was completely reversed after $\omega 3$ treatments. Evidences indicate that transient global ischemia may lead to severe impairments in learning and memory [48-50]. The pyramidal CA1 neurons of the hippocampus are critically involved in spatial learning and memory and are also especially vulnerable to cerebral ischemia. The transient global cerebral ischemia model results in neurodegeneration, known to induce both hippocampal neuronal loss and learning deficits in rats [48, $50,51]$. Reduction of memory deficits by PUFA, after global ischemia, has been already observed by others [30, 34, 52]. However, in most of these data, the animals were chronically subjected to a rich-in- $\omega 3$ fatty acids diet, while in the present study the animals were orally given lower doses of $\omega 3$, for a short period of time.

We also showed a $37 \%$ reduction in DA levels in the striata of ischemic rats, as related to the SO group. On the other hand, these effects were completely reverted after $\omega 3$ treatments and a similar picture was noticed in DOPAC levels. Ischemic conditions can induce the release of brain neurotransmitters. Earlier studies showed that the DA metabolism is significantly altered during and after ischemia in the rabbit retina [53]. On the other hand, biochemical consequences of ischemia in the hippocampus, cortex, and striatum have been object of much attention, because these brain structures are particularly vulnerable to ischemia [54]. The extracellular levels of DA have been shown to increase drastically, immediately after ischemia. This increase is followed by a great decrease, probably reflecting that the release of DA is not compensated by an increased synthesis of this neurotransmitter [55-60].

Evidences indicate that DA release exerts an important role in the striatal damage observed after ischemia. As a matter of fact, the depletion of DA by alpha-methyltyrosine or 6-OHDA before the ischemic process reduces the degree of neuronal damage [61-63], using an experimental model similar to ours, showing by microdialysis that the extracellular concentrations of DA increased abruptly, around $3 \mathrm{~min}$ after the ischemic insult, reaching a maximum value between 20 and $40 \mathrm{~min}$ after the insult and decreasing subsequently. These authors concluded that, during ischemia, the great increase in DA concentrations occurs probably as a result of energy failure in cellular membranes. Then, the DA release could be the cause of the neuronal damage, associated with brain ischemia.

In our study, it is possible that, because DA determinations in the striatum were carried out 7 days after ischemia, this brain tissue was already partly recovered. Despite that, our results showed a partial recovery of the alterations in DA levels in the ischemic groups after $\omega 3$ treatments. 
Others [7] showed that $\omega 3$ fatty acids present neuroprotective properties, exerting benefits on cognitive functions, neurodegenerative diseases, and brain ischemia.

Evidences [64] have indicated the effects of a diet which was deficient in alpha-linolenic acid, precursor of long chains unsaturated fatty acids as $\omega 3$, on the dopaminergic neurotransmission in the core of the rat nucleus accumbens. These in vivo microdialysis experiments showed increased basal levels of dopamine and their metabolites (DOPAC and HVA), in rats subjected to a fatty-acid-deficient diet, as compared to controls, indicating alterations in the dopaminergic neurotransmission in the nucleus accumbens.

For the assessment of neuronal degeneration, we used the staining with Fluoro-Jade which is a fluorescein derivative that specifically binds to degenerating neurons. Fluoro-Jade stains cell bodies, dendrites, axons, and their terminals but does not stain healthy neurons [65]. We showed a high number of degenerating neurons in the CA1, CA3, dentate gyrus, and temporal cortex from untreated ischemic rats, and this profile was completely reversed after $\omega 3$ treatments, towards that of SO (controls), indicating the drug neuroprotective effects. Interestingly, Fluoro-Jade staining demonstrating neuronal damage was shown in some brain areas of animals submitted to PUFAs dietary restriction [66], corroborating with our findings.

While PUFAs are known to present brain immunomodulatory properties, n-3 PUFAs are able to reduce inflammation and n-6 PUFAs are more proinflammatory [67]. These authors showed that the central n-3 PUFA increase modulates the brain innate immune system, leading to the protection against LPS-induced proinflammatory cytokine production and spatial memory impairment, in transgenic mice. Recently [68], $\omega 3$ was shown to reduce cytokine-induced expression of proatherogenic and proinflammatory proteins, in human endothelial cells, and these properties may contribute to the antiatherogenic and anti-inflammatory effects of n-3 PUFAs.

In the present study, the treatment of ischemic animals with $\omega 3$ drastically decreased immunoreactivity for TNFalpha in the hippocampus and temporal cortex. A previous work [69] demonstrated that $\omega 3$ pretreatments significantly attenuated LPS-stimulated TNF-alpha production. Others [70] showed that the inhibition by $\omega 3$ of proinflammatory cytokines as TNF-alpha, in murine macrophages, is in part mediated by the inactivation of NF-kappaB signaling. Besides, Nielsen et al., 2005 [71], observed that $\omega 3$ fatty acids were able to inhibit the increase in proinflammatory cytokines, in patients with Crohn's disease. $\omega 3$ fatty acids are known to affect immune response, partly by influencing cytokine secretion [72].

Furthermore, we also showed that the treatments with $\omega 3$ of ischemic animals significantly decreased COX-2 and iNOS immunoreactivity in hippocampal brain areas. Evidences indicate that $\omega 3$ fatty acids dampen inflammation, through multiple pathways, and directly or indirectly suppress the activity of nuclear transcription factors, as NF-kappaB, and reduce the production of proinflammatory enzymes and cytokines, including COX-2, TNF-alpha, and IL-1B [73]. NFkappaB is one of the most important transcription factors involved in inflammatory response and upregulation of gene encoding of inflammatory cytokines, adhesion molecules, and COX-2. Evidences indicated that $\omega 3$ decreases expression of adhesion molecules and production of inflammatory cytokines and COX-2 metabolites, and a common mechanism would be the impact on the NFkB system [74]. This could well be the way $\omega 3$ is acting in our study.

Besides decreasing COX-2 immunostaining, $\omega 3$ attenuates hippocampal iNOS immunoreactivity. It has been shown that a diet rich in $\omega 3$ fatty acids decreases eNOS and iNOS in the diabetic kidney [75]. Interestingly, the attenuation of iNOS in LPS-stimulated macrophages by $\omega 3$ was shown to be independent of COX-2 derived PGE2, contradicting the hypothesis that the decrease in NO production associated with $\omega 3$ treatments occurs through a COX-2 derived PGE2 dependent mechanism [76]. The expression of iNOS is one direct consequence of inflammatory processes [77]. One important step during inflammation is leukocyte infiltration, mainly controlled by chemokines. The production of these chemokines is positively or negatively regulated by iNOSderived NO [78]. Furthermore, a clinical study demonstrated that fish oil decreased serum levels of TNF-alpha, IL1beta, IL-6, and nitric oxide metabolites in multiple sclerosis patients treated with IFN-1beta [79].

\section{Conclusion}

$\omega 3$ fatty acids by their anti-inflammatory and antioxidant properties that contribute to the neuroprotective property might be a therapeutic target to be pursued for the prevention or treatment of inflammation-related diseases. $\omega 3$ deficiency increases the brain's vulnerability, representing an important risk factor for the development of neuropathologies [80]. Furthermore, understanding the precise roles of $\omega 3$ fatty acids, as a possible disease modifier, will permit the development of new therapies for neurological diseases.

\section{Competing Interests}

The authors declare no conflict of interests.

\section{Authors' Contributions}

Maria Elizabeth Pereira Nobre supervised the experimental protocol; Alyne Oliveira Correia, Francisco Nilson Maciel Mendonça, and Luiz Ricardo Araújo Uchoa performed the brain ischemia experiments; Jessica Tamara Nunes Vasconcelos and Carlos Ney Alencar de Araújo carried out the daily animals treatments; Gerly Anne de Castro Brito supervised the immunohistochemical assays; Rafaelly Maria Pinheiro Siqueira, Gilberto dos Santos Cerqueira, and Kelly Rose Tavares Neves performed the immunohistochemical assays; Ricardo Mário Arida and Glauce Socorro de Barros Viana were responsible for the design, statistical analysis, and draft of the paper. All authors read and approved the final version of the paper.

\section{Acknowledgments}

The authors thank the Brazilian National Research Council (CNPq) and the Foundation for Research and Development 
(FUNCAP) of the Ceará State, Brazil, for financial support; Maria Vilani Rodrigues Bastos for her technical assistance; and Professor M. O. L. Viana for the orthographic revision of the paper.

\section{References}

[1] A. Durukan and T. Tatlisumak, "Acute ischemic stroke: overview of major experimental rodent models, pathophysiology, and therapy of focal cerebral ischemia," Pharmacology Biochemistry and Behavior, vol. 87, no. 1, pp. 179-197, 2007.

[2] S. Braeuninger and C. Kleinschnitz, "Rodent models of focal cerebral ischemia: procedural pitfalls and translational problems," Experimental \& Translational Stroke Medicine, vol. 1, pp. 1-11, 2009.

[3] M. Bacigaluppi, G. Comi, and D. M. Hermann, "Animal models of ischemic stroke. part two: modeling cerebral ischemia," The Open Neurology Journal, vol. 4, pp. 34-38, 2010.

[4] Y. Huang and J. O. McNamara, "Ischemic stroke: 'Acidotoxicity' is a perpetrator," Cell, vol. 118, no. 6, pp. 665-666, 2004.

[5] P. Mergenthaler, U. Dirnagl, and A. Meisel, "Pathophysiology of stroke: lessons from animal models," Metabolic Brain Disease, vol. 19, no. 3-4, pp. 151-167, 2004.

[6] T. G. Phan, P. M. Wright, R. Markus, D. W. Howells, S. M. Davis, and G. A. Donnan, "Salvaging the ischaemic penumbra: more than just reperfusion?" Clinical and Experimental Pharmacology and Physiology, vol. 29, no. 1-2, pp. 1-10, 2002.

[7] W. Zhang, P. Li, X. Hu, F. Zhang, J. Chen, and Y. Gao, "Omega3 polyunsaturated fatty acids in the brain: metabolism and neuroprotection," Frontiers in Bioscience, vol. 16, no. 7, pp. 26532670, 2011.

[8] J. W. Phillis and M. H. O'Regan, "Characterization of modes of release of amino acids in the ischemic/reperfused rat cerebral cortex," Neurochemistry International, vol. 43, no. 4-5, pp. 461467, 2003.

[9] S. Chalon, S. Delion-Vancassel, C. Belzung et al., "Dietary fish oil affects monoaminergic neurotransmission and behavior in rats," Journal of Nutrition, vol. 128, no. 12, pp. 2512-2519, 1998.

[10] S. Chalon, "Omega-3 fatty acids and monoamine neurotransmission," Prostaglandins Leukotrienes and Essential Fatty Acids, vol. 75, no. 4-5, pp. 259-269, 2006.

[11] C. J. S. Price, E. A. Warburton, and D. K. Menon, "Human cellular inflammation in the pathology of acute cerebral ischaemia," Journal of Neurology, Neurosurgery and Psychiatry, vol. 74, no. 11, pp. 1476-1484, 2003.

[12] R. Jin, G. Yang, and G. Li, "Inflammatory mechanisms in ischemic stroke: role of inflammatory cells," Journal of Leukocyte Biology, vol. 87, no. 5, pp. 779-789, 2010.

[13] P. C. Calder, "Dietary modification of inflammation with lipids," Proceedings of the Nutrition Society, vol. 61, no. 3, pp. 345-358, 2002.

[14] A. P. Simopoulos, "Omega-3 fatty acids in inflammation and autoimmune diseases," Journal of the American College of Nutrition, vol. 21, no. 6, pp. 495-505, 2002.

[15] P. C. Calder, "Omega-3 fatty acids and inflammatory processes," Nutrients, vol. 2, no. 3, pp. 355-374, 2010.

[16] P. C. Calder, "Fatty acids and inflammation: the cutting edge between food and pharma," European Journal of Pharmacology, vol. 668, no. 1, pp. S50-S58, 2011.

[17] M. E. P. Nobre, A. O. Correia, M. B. Borges et al., "Eicosapentaenoic acid and docosahexaenoic acid exert anti-inflammatory and antinociceptive effects in rodents at low doses," Nutrition Research, vol. 34, pp. 251-263, 2013.

[18] L. Prut and C. Belzung, "The open field as a paradigm to measure the effects of drugs on anxiety-like behaviors: a review," European Journal of Pharmacology, vol. 463, no. 1-3, pp. 3-33, 2003.

[19] R. G. M. Morris, "Spatial localization does not require the presence of local cues," Learning and Motivation, vol. 12, no. 2, pp. 239-260, 1981.

[20] C. V. Vorhees and M. T. Williams, "Morris water maze: procedures for assessing spatial and related forms of learning and memory," Nature Protocols, vol. 1, no. 2, pp. 848-858, 2006.

[21] J. B. Bederson, L. H. Pitts, S. M. Germano, M. C. Nishimura, R. L. Davis, and H. M. Bartkowski, "Evaluation of 2,3,5-triphenyltetrazolium chloride as a stain for detection and quantification of experimental cerebral infarction in rats," Stroke, vol. 17, no. 6 , pp. 1304-1308, 1986.

[22] F. Block, "Global ischemia and behavioural deficits," Progress in Neurobiology, vol. 58, no. 3, pp. 279-295, 1999.

[23] H. Hodges, A. Nelson, D. Virley, T. R. Kershaw, and J. D. Sinden, "Cognitive deficits induced by global cerebral ischaemia: prospects for transplant therapy," Pharmacology Biochemistry and Behavior, vol. 56, no. 4, pp. 763-780, 1997.

[24] W. A. Pulsinelli, J. B. Brierley, and F. Plum, "Temporal profile of neuronal damage in a model of transient forebrain ischemia," Annals of Neurology, vol. 11, no. 5, pp. 491-498, 1982.

[25] M. A. Atlasi, H. Naderian, M. Noureddini, E. Fakharian, and A. Azami, "The morphology of rat hippocampus CA1 neurons following modified two and four vessel global ischemia models," Archives of Trauma Research, vol. 2, no. 3, pp. 124-128, 2013.

[26] I. Fedorova and N. Salem Jr., "Omega-3 fatty acids and rodent behavior," Prostaglandins Leukotrienes and Essential Fatty Acids, vol. 75, no. 4-5, pp. 271-289, 2006.

[27] T. Takeuchi, M. Iwanaga, and E. Harada, "Possible regulatory mechanism of DHA-induced anti-stress reaction in rats," Brain Research, vol. 964, no. 1, pp. 136-143, 2003.

[28] A. Harauma and T. Moriguchi, "Dietary n-3 fatty acid deficiency in mice enhances anxiety induced by chronic mild stress," Lipids, vol. 46, no. 5, pp. 409-416, 2011.

[29] N. Vinot, M. Jouin, A. Lhomme-Duchadeuil et al., "Omega3 fatty acids from fish oil lower anxiety, improve cognitive functions and reduce spontaneous locomotor activity in a nonhuman primate," PLoS ONE, vol. 6, no. 6, Article ID e20491, 2011.

[30] H. Plamondon and M.-C. Roberge, "Dietary PUFA supplements reduce memory deficits but not CA1 ischemic injury in rats," Physiology and Behavior, vol. 95, no. 3, pp. 492-500, 2008.

[31] H.-M. Su, "Mechanisms of n-3 fatty acid-mediated development and maintenance of learning memory performance," Journal of Nutritional Biochemistry, vol. 21, no. 5, pp. 364-373, 2010.

[32] M. Hashimoto, S. Hossain, Y. Tanabe et al., "The protective effect of dietary eicosapentaenoic acid against impairment of spatial cognition learning ability in rats infused with amyloid $\beta_{(1-40)}$," Journal of Nutritional Biochemistry, vol. 20, no. 12, pp. 965-973, 2009.

[33] S. C. Heinrichs, "Dietary $\omega 3$ fatty acid supplementation for optimizing neuronal structure and function," Molecular Nutrition and Food Research, vol. 54, no. 4, pp. 447-456, 2010.

[34] J. S. Fernandes, M. A. Mori, R. Ekuni, R. M. W. Oliveira, and H. Milani, "Long-term treatment with fish oil prevents memory impairments but not hippocampal damage in rats subjected to 
transient, global cerebral ischemia," Nutrition Research, vol. 28, no. 11, pp. 798-808, 2008.

[35] N. Okabe, T. Nakamura, T. Toyoshima, O. Miyamoto, F. Lu, and T. Itano, "Eicosapentaenoic acid prevents memory impairment after ischemia by inhibiting inflammatory response and oxidative damage," Journal of Stroke and Cerebrovascular Diseases, vol. 20, no. 3, pp. 188-195, 2011.

[36] N. F. A. Sopian, M. Ajat, N. I. Shafie et al., "Does shortterm dietary omega-3 fatty acid supplementation influence brain hippocampus gene expression of zinc transporter-3?” International Journal of Molecular Sciences, vol. 16, no. 7, pp. 15800-15810, 2015.

[37] L. G. Puskás, K. Kitajka, C. Nyakas, G. Barcelo-Coblijn, and T. Farkas, "Short-term administration of omega 3 fatty acids from fish oil results in increased transthyretin transcription in old rat hippocampus," Proceedings of the National Academy of Sciences of the United States of America, vol. 100, no. 4, pp. 1580-1585, 2003.

[38] W. Zhang, X. Hu, W. Yang, Y. Gao, and J. Chen, "Omega3 polyunsaturated fatty acid supplementation confers longterm neuroprotection against neonatal hypoxic-ischemic brain injury through anti-inflammatory actions," Stroke, vol. 41, no. 10, pp. 2341-2347, 2010.

[39] X. Tang, Z.-J. Li, J. Xu et al., "Short term effects of different omega-3 fatty acid formulation on lipid metabolism in mice fed high or low fat diet," Lipids in Health and Disease, vol. 11, article 70, 2012.

[40] M. Ajami, S. Eghtesadi, R. Habibey et al., "Effect of short- and long-term treatment with Omega-3 fatty acids on scopolamineinduced amnésia," Iranian Journal of Pharmaceutical Research, vol. 11, no. 2, pp. 533-540, 2012.

[41] S. K. Raatz, J. B. Redmon, N. Wimmergren, J. V. Donadio, and D. M. Bibus, "Enhanced absorption of n-3 fatty acids from emulsified compared with encapsulated fish oil," Journal of the American Dietetic Association, vol. 109, no. 6, pp. 1076-1081, 2009.

[42] K. Yurko-Mauro, D. McCarthy, D. Rom et al., "Beneficial effects of docosahexaenoic acid on cognition in age-related cognitive decline," Alzheimer's and Dementia, vol. 6, no. 6, pp. 456-464, 2010.

[43] S. C. Gerhardt and C. A. Boast, "Motor activity changes following cerebral ischemia in gerbils are correlated with the degree of neuronal degeneration in hippocampus," Behavioral Neuroscience, vol. 102, no. 2, pp. 301-303, 1988.

[44] Y. Karasawa, H. Araki, and S. Otomo, "Changes in locomotor activity and passive avoidance task performance induced by cerebral ischemia in mongolian gerbils," Stroke, vol. 25, no. 3, pp. 645-650, 1994.

[45] M. Milot and H. Plamondon, "Ischemia-induced hyperactivity: effects of dim versus bright illumination on open-field exploration and habituation following global ischemia in rats," Behavioural Brain Research, vol. 192, no. 2, pp. 166-172, 2008.

[46] M. R. Milot and H. Plamondon, "Time-dependent effects of global cerebral ischemia on anxiety, locomotion, and habituation in rats," Behavioural Brain Research, vol. 200, no. 1, pp. 173$180,2009$.

[47] T. G. Ohk, K.-Y. Yoo, S. M. Park et al., "Neuronal damage using fluoro-jade B histofluorescence and gliosis in the striatum after various durations of transient cerebral ischemia in gerbils," Neurochemical Research, vol. 37, no. 4, pp. 826-834, 2012.

[48] R. E. Hartman, J. M. Lee, G. J. Zipfel, and D. F. Wozniak, "Characterizing learning deficits and hippocampal neuron loss following transient global cerebral ischemia in rats," Brain Research, vol. 1043, no. 1-2, pp. 48-56, 2005.

[49] M. Von Euler, O. Bendel, T. Bueters, J. Sandin, and G. Von Euler, "Profound but transient deficits in learning and memory after global ischemia using a novel water maze test," Behavioural Brain Research, vol. 166, no. 2, pp. 204-210, 2006.

[50] K. D. Langdon, S. Granter-Button, and D. Corbett, "Persistent behavioral impairments and neuroinflammation following global ischemia in the rat," European Journal of Neuroscience, vol. 28, no. 11, pp. 2310-2318, 2008.

[51] T. Bueters, M. von Euler, O. Bendel, and G. von Euler, "Degeneration of newly formed CA1 neurons following global ischemia in the rat," Experimental Neurology, vol. 209, no. 1, pp. 114-124, 2008.

[52] C. Luo, H. Ren, J.-B. Wan et al., "Enriched endogenous omega3 fatty acids in mice protect against global ischemia injury," Journal of Lipid Research, vol. 55, no. 7, pp. 1288-1297, 2014.

[53] W. Cao, A. Drumheller, M. Zaharia, G. Lafond, J.-R. Brunette, and F. B. Jolicoeur, "Effects of experimentally induced ischemia on dopamine metabolism in rabbit retina," Investigative Ophthalmology \& Visual Science, vol. 34, no. 11, pp. 3140-3146, 1993.

[54] W. A. Pulsinelli and T. E. Duffy, "Regional energy balance in rat brain after transient forebrain ischemia," Journal of Neurochemistry, vol. 40, no. 5, pp. 1500-1503, 1983.

[55] L. A. Phebus and J. A. Clemens, "Effects of transient, global, cerebral ischemia on striatal extracellular dopamine, serotonin and their metabolites," Life Sciences, vol. 44, no. 19, pp. 1335$1342,1989$.

[56] M. Y.-T. Globus, "Role of dopamine in ischemic neuronal damage," Stroke, vol. 20, no. 6, pp. 827-828, 1989.

[57] T. Kawano, K. Tsutsumi, H. Miyake, and K. Mori, "Striatal dopamine in acute cerebral ischemia of stroke-resistant rats," Stroke, vol. 19, no. 12, pp. 1540-1543, 1988.

[58] A. Slivka, T. S. Brannan, J. Weinberger, P. J. Knott, and G. Cohen, "Increase in extracellular dopamine in the striatum during cerebral ischemia: a study utilizing cerebral microdialysis," Journal of Neurochemistry, vol. 50, no. 6, pp. 1714-1718, 1988.

[59] A. Kuruvilla, R. Cherian, D. R. Theodore, and J. Abraham, "Temporal profile of tissue levels of dopamine and its metabolites, HVA and DOPAC following focal cerebral ischaemia in anaesthetized primates," Clinical and Experimental Pharmacology and Physiology, vol. 13, no. 7, pp. 519-524, 1986.

[60] K. Ogura, M. Shibuya, Y. Suzuki, M. Kanamori, and I. Ikegaki, "Changes in striatal dopamine metabolism measured by in vivo voltammetry during transient brain ischemia in rats," Stroke, vol. 20, no. 6, pp. 783-787, 1989.

[61] T. Brannan, J. Weinberger, P. Knott et al., "Direct evidence of acute, massive striatal dopamine release in gerbils with unilateral strokes," Stroke, vol. 18, no. 1, pp. 108-110, 1987.

[62] J. Weinberger, J. Nieves-Rosa, and G. Cohen, "Nerve terminal damage in cerebral ischemia: protective effect of alpha-methylpara-tyrosine," Stroke, vol. 16, no. 5, pp. 864-870, 1985.

[63] J. A. Clemens and L. A. Phebus, "Dopamine depletion protects striatal neurons from ischemia-induced cell death," Life Sciences, vol. 42, no. 6, pp. 707-713, 1988.

[64] L. Zimmer, S. Delion-Vancassel, G. Durand et al., "Modification of dopamine neurotransmission in the nucleus accumbens of rats deficient in n-3 polyunsaturated fatty acids," Journal of Lipid Research, vol. 41, no. 1, pp. 32-40, 2000.

[65] L. C. Schmued and K. J. Hopkins, "Fluoro-Jade B: a high affinity fluorescent marker for the localization of neuronal degeneration," Brain Research, vol. 874, no. 2, pp. 123-130, 2000. 
[66] H. D. Cardoso, P. P. Passos, C. J. Lagranha et al., "Differential vulnerability of Substantia nigra and Corpus striatum to oxidative insult induced by reduced dietary levels of essential fatty acids," Frontiers in Human Neuroscience, vol. 6, article 249, 2012.

[67] J.-C. Delpech, C. Madore, C. Joffre et al., "Transgenic increase in $n-3 / n-6$ fatty acid ratio protects against cognitive deficits induced by an immune challenge through decrease of neuroinflammation," Neuropsychopharmacology, vol. 40, no. 3, pp. 525536, 2015.

[68] R. De Caterina, M. I. Cybulsky, S. K. Clinton, M. A. Gimbrone Jr., and P. Libby, "The omega-3 fatty acid docosahexaenoate reduces cytokine-induced expression of proatherogenic and proinflammatory proteins in human endothelial cells," Arteriosclerosis, Thrombosis, and Vascular Biology, vol. 14, no. 11, pp. 1829-1836, 1994.

[69] T. A. Babcock, W. S. Helton, D. Hong, and N. J. Espat, "Omega3 fatty acid lipid emulsion reduces LPS-stimulated macrophage TNF- $\alpha$ production," Surgical Infections, vol. 3, no. 2, pp. 145-149, 2002.

[70] T. E. Novak, T. A. Babcock, D. H. Jho, W. S. Helton, and N. J. Espat, "NF- $\kappa$ B inhibition by $\omega-3$ fatty acids modulates LPSstimulated macrophage TNF- $\alpha$-transcription," American Journal of Physiology-Lung Cellular and Molecular Physiology, vol. 284, no. 1, pp. L84-L89, 2003.

[71] A. A. Nielsen, L. G. M. Jørgensen, J. N. Nielsen et al., "Omega3 fatty acids inhibit an increase of proinflammatory cytokines in patients with active Crohn's disease compared with omega- 6 fatty acids," Alimentary Pharmacology and Therapeutics, vol. 22, no. 11-12, pp. 1121-1128, 2005.

[72] I. H. Skuladottir, D. H. Petursdottir, and I. Hardardottir, "The effects of omega- 3 polyunsaturated fatty acids on TNF- $\alpha$ and IL-10 secretion by murine peritoneal cells in vitro," Lipids, vol. 42, no. 8, pp. 699-706, 2007.

[73] J. X. Kang and K. H. Weylandt, "Modulation of inflammatory cytokines by omega-3 fatty acids," Sub-Cellular Biochemistry, vol. 49, pp. 133-143, 2008.

[74] P. C. Calder, "Omega-3 polyunsaturated fatty acids and inflammatory processes: nutrition or pharmacology?" British Journal of Clinical Pharmacology, vol. 75, no. 3, pp. 645-662, 2013.

[75] J. H. Garman, S. Mulroney, M. Manigrasso, E. Flynn, and C. Maric, "Omega-3 fatty acid rich diet prevents diabetic renal disease," American Journal of Physiology-Renal Physiology, vol. 296, no. 2, pp. F306-F316, 2009.

[76] A. Razzak, C. Aldrich, T. A. Babcock, A. Saied, and N. J. Espat, "Attenuation of iNOS in an LPS-stimulated macrophage model by omega-3 fatty acids is independent of COX-2 derived PGE2," Journal of Surgical Research, vol. 145, no. 2, pp. 244-250, 2008.

[77] C. Suschek, O. Schnorr, and V. Kolb-Bachofen, "The role of iNOS in chronic inflammatory processes in vivo: Is it damagepromoting, protective, or active at all?" Current Molecular Medicine, vol. 4, no. 7, pp. 763-775, 2004.

[78] Y. Kobayashi, "The regulatory role of nitric oxide in proinflammatory cytokine expression during the induction and resolution of inflammation," Journal of Leukocyte Biology, vol. 88, no. 6, pp. 1157-1162, 2010.

[79] V. Ramirez-Ramirez, M. A. Macias-Islas, G. G. Ortiz et al., "Efficacy of fish oil on serum of TNF $\alpha$, IL- $1 \beta$, and IL- 6 oxidative stress markers in multiple sclerosis treated with interferon beta1b," Oxidative Medicine and Cellular Longevity, vol. 2013, Article ID 709493, 8 pages, 2013.

[80] N. Blondeau, R. H. Lipsky, M. Bourourou, M. W. Duncan, P. B. Gorelick, and A. M. Marini, "Alpha-linolenic acid: an omega-3 fatty acid with neuroprotective properties-Ready for use in the stroke clinic?" BioMed Research International, vol. 2015, Article ID 519830, 8 pages, 2015. 


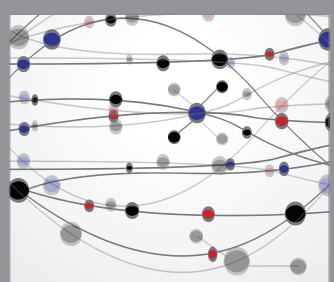

The Scientific World Journal
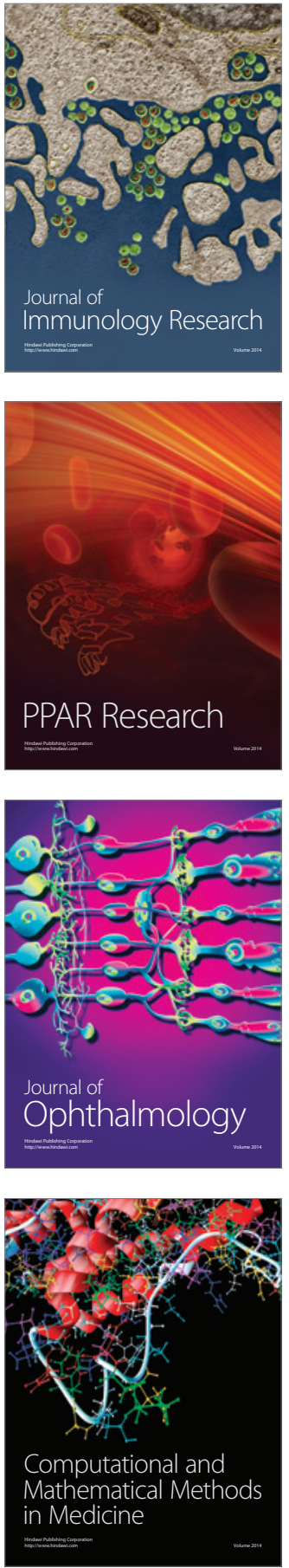

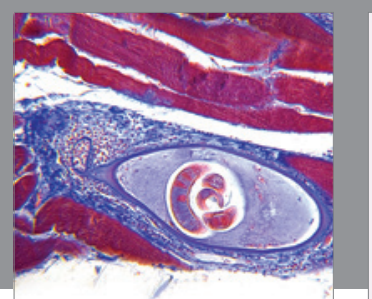

Gastroenterology Research and Practice

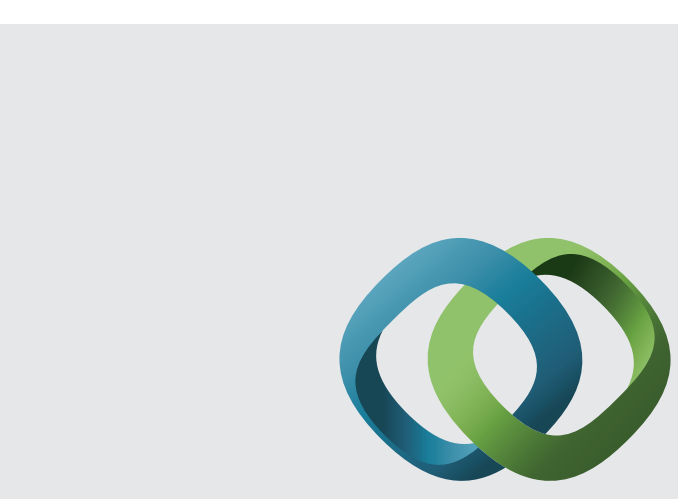

\section{Hindawi}

Submit your manuscripts at

http://www.hindawi.com
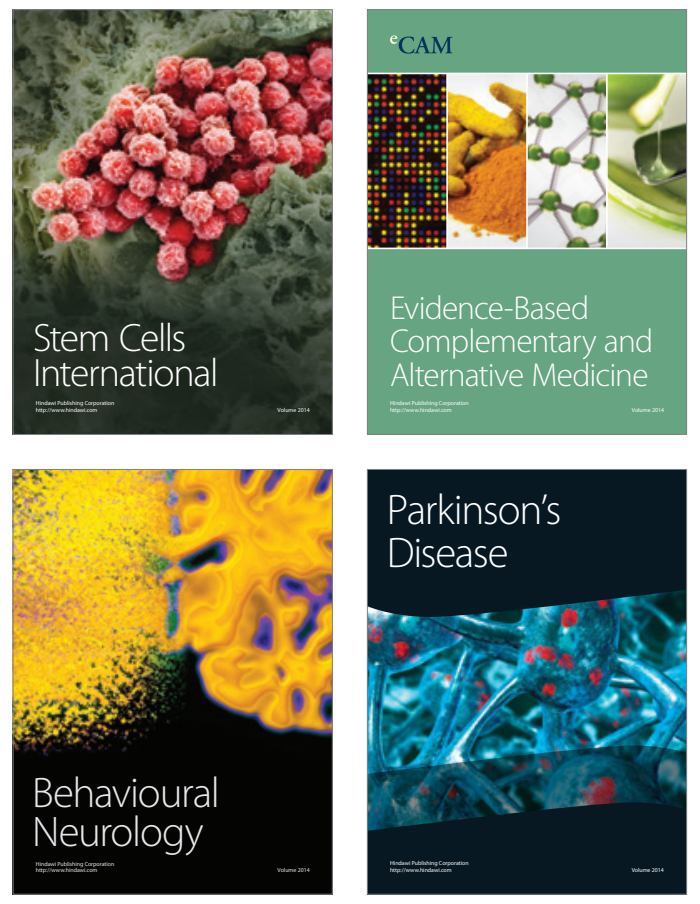
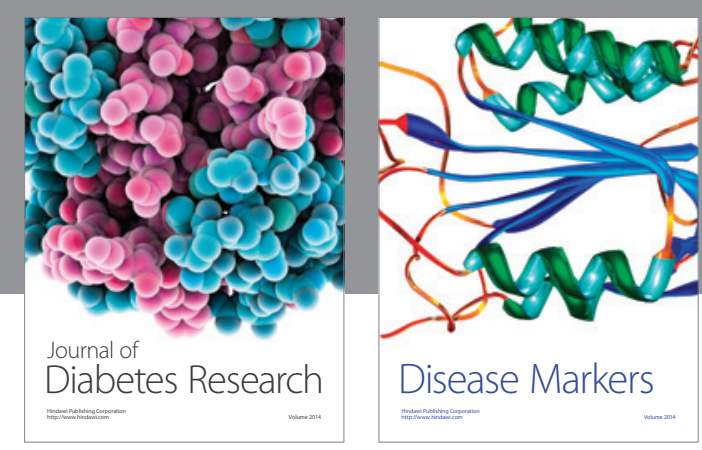

Disease Markers
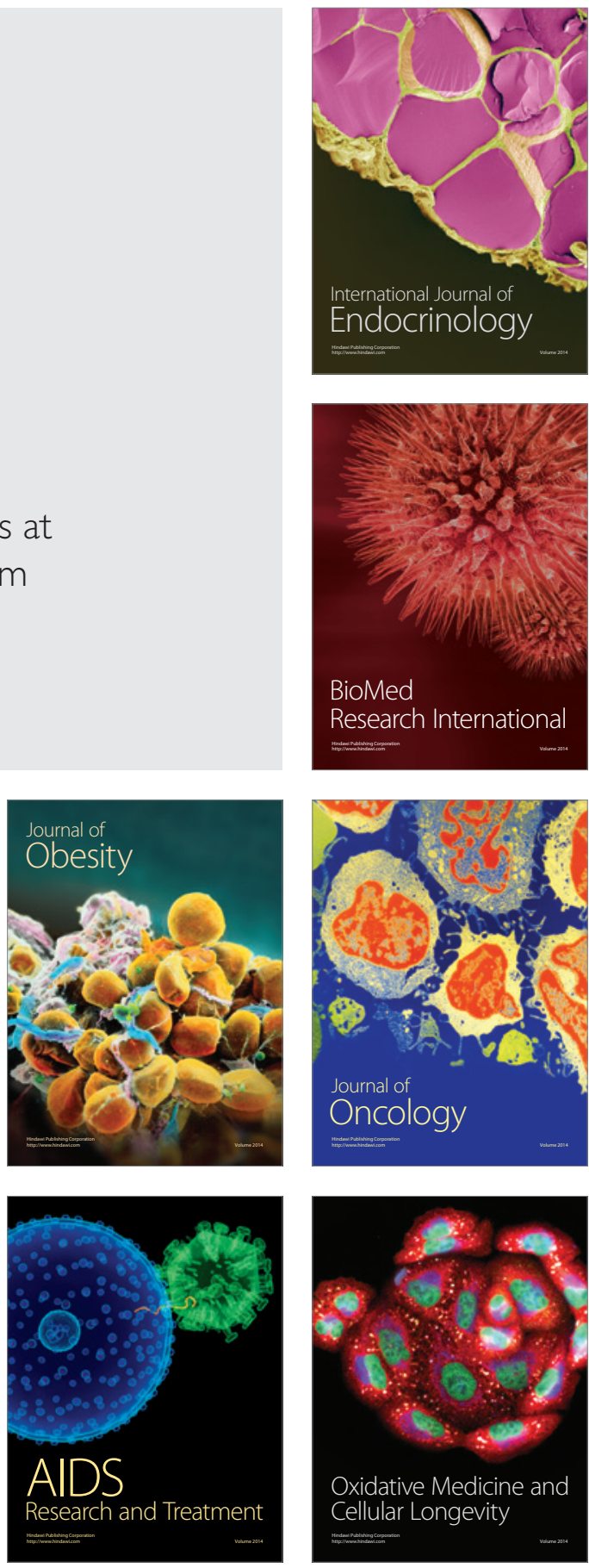